\title{
MPC for tracking periodic reference signals
}

\author{
D. Limon* T. Alamo* D.Muñoz de la Peña* \\ M.N. Zeilinger ${ }^{* *}$ C.N. Jones ${ }^{* *}$ M. Pereira ${ }^{*}$ \\ * Departamento de Ingeniería de Sistemas y Automática, Escuela \\ Superior de Ingenieros, Universidad de Sevilla. Sevilla, Spain. (e-mail: \\ \{limon,alamo,davidmps\}@cartuja.us.es) \\ ** Automatic Control Laboratory. EPFL. Lausanne, Switzerland. \\ (e-mail: \{melanie.zeilinger,colin.jones\}@epfl.ch)
}

\begin{abstract}
This paper is devoted to the design of a predictive controller for constrained linear systems to track periodic references. The only assumption on the dynamics of the reference is that it is periodic and its period is known. It is also assumed that the reference signal is a priori known by the controller. Inspired in the hierarchical control scheme based on the trajectory planification, the ideas of the MPC for tracking [Limon et al., 2008] are extended to this case. The proposed predictive controller has the future sequence of inputs and an artificial reference as decision variables. The cost function is divided into two terms: one penalizes the tracking error with the artificial reference and other penalizes the deviation of the artificial reference to the reference to be tracked. Stability is ensured thanks to the addition of two constraints: a terminal constraint on the predicted trajectory and a constraint that enforces the artificial reference to be periodic. It is proved that the proposed controller is recursively feasible and the controlled system satisfies the hard constraints, is asymptotically stable and converges to the best possible reachable trajectory. The properties of the proposed controller are illustrated in an example.
\end{abstract}

\section{INTRODUCTION}

Model predictive control is one of the few control techniques capable of regulating the controlled variable ensuring constraint satisfaction. For a given set-point, the MPC can be suitably designed to ensure closed-loop stability and constraint satisfaction. However if the set-point changes, the stabilizing design of the predictive controller may not be valid anymore and/or feasibility of the controller may be lost [Rawlings and Mayne, 2009].

In order to overcome this problem several solutions have been proposed [Camacho and Bordons, 2004, Rossiter et al., 1996, Chisci and Zappa, 2003]. In [Pannocchia and Kerrigan, 2005, Pannocchia, 2004] the set-point change is considered equivalent to a disturbance to be rejected and asymptotic stability and offset-free is ensured by integrating a disturbance model in the prediction model. A different approach has been proposed in the context of reference governors [Gilbert et al., 1999, Bemporad et al., 1997]. This control technique assumes that the system is robustly stabilized by a local controller, and a nonlinear filtering of the reference is designed to ensure robust satisfaction of the constraints. These controllers ensure robust tracking without considering the performance of the obtained controller nor the domain of attraction.

In [Limon et al., 2008, Ferramosca et al., 2009] an MPC for tracking constant references is proposed, which is able to lead the system to any admissible set point in an admissible way. The main characteristics of this controller are: an

\footnotetext{
* This work has been funded by the National Plan Projects DPI2008-05818 and DPI2010-21589-C05-01 of the Spanish Ministry of Science and Innovation and FEDER funds.
}

artificial steady state is considered as a decision variable, a cost that penalizes the error with the artificial steady state is minimized, an additional term that penalizes the deviation between the artificial steady state and the target steady state is added to the cost function (the so-called offset cost function) and an invariant set for tracking is considered as extended terminal constraint. This controller ensures that under any change of the target steady state, the closed loop system maintains the feasibility of the controller and ensures the convergence to the target if admissible.

For the case of non-constant references, in [Magni et al., 2001] a class of output feedback MPC for nonlinear discrete-time systems is proposed to solve the problem of tracking exogenous signals (and asymptotically rejecting disturbances) generated by systems with a known dynamics. In [Mäder and Morari, 2010] the authors consider the problem of offset-free tracking for reference signals generated by arbitrary dynamics, excluding the consideration of feasibility and stability of the closed-loop system.

Periodic systems are important extensions of linear timeinvariant systems and allow modeling of important control problems [Bittanti and Colaneri, 2009, Varga, 2007, Lovera and Varga, 2005]. Available methods for tracking based on MPC generally consider the case of (piecewise) constant references. The problem of tracking periodic references for LTI systems can, however, be considered as the problem of tracking constant references of periodic systems using a different modeling framework. MPC of discrete-time linear/nonlinear periodic systems was tackled, e.g., in [Böhm et al., 2009, Kern et al., 2009, Kim et al., 2000, Lee et al., 2001, Gondhalekar and Jones, 2011]. 
In this paper, the tracking problem of periodic references is considered, whose future evolution is assumed to be known but not their dynamics. This problem is analyzed for constrained linear systems and, based on the ideas of [Limon et al., 2008], a novel MPC controller is proposed. As in [Limon et al., 2008], an artificial reachable reference is considered as decision variable as well as the predicted input of the system, a cost function that penalizes the tracking error between the predicted trajectory and the artificial reference is considered, an extended terminal constraint is added and a term penalizing the deviation between the artificial reference trajectory and the known future reference is also added. The proposed controller ensures an admissible evolution for any possible reference and asymptotic stability to the best reachable reference. Then if the reference is reachable by the constrained system, this reference will be asymptotically tracked without error.

\section{PROBLEM STATEMENT AND CONTROL OBJECTIVE}

Consider a linear time-invariant system described by a state-space linear model

$$
\begin{aligned}
x(k+1) & =A x(k)+B u(k) \\
y(k) & =C x(k)+D u(k)
\end{aligned}
$$

where $x(k) \in \mathbb{R}^{n}$ is the state at the sampling time $k$, $u(k) \in \mathbb{R}^{m}$ is the manipulable input and $y(k) \in \mathbb{R}^{p}$ is the controlled variables. It is assumed that $(A, B)$ is controllable.

The evolution of the plant must be such that the following constraints must hold

$$
(x(k), u(k)) \in \mathcal{Z}
$$

where set $\mathcal{Z}$ is a convex and closed polyhedron.

The control objective is to track an exogenous and apriori known reference signal $\mathbf{r}=\{r(0), r(1), \cdots\}$ for the controlled output $y(k)$. The reference is assumed to be periodic, that is $r\left(k+N_{r}\right)=r(k)$ for all $k \geq 0$, and the period of the signal $N_{r}$ is known. Then a control law $u(k)=\kappa(x(k), \mathbf{r})$ must be designed to ensure that the controlled system

$$
\begin{array}{r}
x(k+1)=A x(k)+B \kappa(x(k), \mathbf{r}) \\
y(k)=C x(k)+D \kappa(x(k), \mathbf{r})
\end{array}
$$

satisfies the constraints along its evolution and if possible, $\lim _{k \rightarrow \infty}\|y(k)-r(k)\|=0$.

In the sequel, for a certain signal $\mathbf{r}=\{\mathbf{r}(\mathbf{0}), \mathbf{r}(\mathbf{1}), \cdots\}, \mathbf{r}_{k}$ will denote $\mathbf{r}_{k}=\left\{r(k), r(k+1), \cdots, r\left(k+N_{r}-1\right)\right\}$.

\section{TRAJECTORY PLANNING AND MPC}

In the tracking problem, the provided reference signal may be impossible to be tracked due to the limits introduced by the constraints or due to a possible inconsistency with the dynamics of the system. In any of these cases, the reference is said to be unreachable. In the following definition the set of reachable trajectories, that is, the set of references that can be tracked, are stated.
Definition 1. A signal $\mathbf{r}$ is said to be reachable if there exist state and input signals $\mathbf{x}$ and $\mathbf{u}$ such that

(1) $(\mathbf{x}, \mathbf{u})$ are coherent with the model (1) for all $k \geq 0$,

(2) $(\mathbf{x}, \mathbf{u})$ are admissible, that is, $(x(k), u(k)) \in \mathcal{Z}$,

(3) $(\mathbf{x}, \mathbf{u})$ maps the reference, that is, $r(k)=C x(k)+D u(k)$

Then, it is clear that the control objective can only be achieved iff the reference signal $\mathbf{r}$ is (or converges to) a reachable signal. If the reference to be tracked is not reachable (which is not unusual in practice), the control problem can be solved by means of a two-layer structure. In the upper level, a trajectory planner calculates a reachable reference $\mathbf{y}^{r}$ as close as possible to the exogenous reference signal $\mathbf{r}$ by solving the following optimization problem:

$$
\begin{aligned}
\left(\mathbf{x}^{r}(k), \mathbf{u}^{r}(k)\right)=\arg \min _{\overline{\mathbf{x}}^{r}, \overline{\mathbf{u}}^{r}} & V_{p}\left(\mathbf{r}_{k} ; \overline{\mathbf{x}}^{r}, \overline{\mathbf{u}}^{r}\right) \\
= & \sum_{i=0}^{N_{r}-1}\left\|\bar{y}^{r}(i)-r(k+i)\right\|_{T}^{2} \\
\text { s.t. } \quad & \bar{x}^{r}(i+1)=A \bar{x}^{r}(i)+B \bar{u}^{r}(i) \\
& \bar{y}^{r}(i)=C \bar{x}^{r}(i)+D \bar{u}^{r}(i) \\
& \left(\bar{x}^{r}(i), \bar{u}^{r}(i)\right) \in \mathcal{Z}
\end{aligned}
$$

Notice that this optimization problem could be calculated off-line due to the periodic nature of the signal.

In the lower level, a predictive controller is designed to track the calculated reachable reference $\left(\mathbf{x}^{r}(k), \mathbf{u}^{r}(k)\right)$ in an admissible way. This is derived from the following optimization problem:

$$
\begin{aligned}
\min _{\overline{\mathbf{u}}} & V\left(x, \mathbf{x}^{r}(k), \mathbf{u}^{r}(k) ; \overline{\mathbf{u}}\right) \\
& =\sum_{i=0}^{N-1}\left\|\bar{y}(i)-\bar{y}^{r}(i)\right\|_{Q}^{2}+\left\|\bar{u}(i)-u^{r}(i \mid k)\right\|_{R}^{2} \\
\text { s.t. } & \bar{x}(i+1)=A \bar{x}(i)+B \bar{u}(i) \\
& \bar{x}(0)=x(k) \\
& \bar{y}(i)=C \bar{x}(i)+D \bar{u}(i) \\
& \bar{y}^{r}(i)=C x^{r}(i \mid k)+D u^{r}(i \mid k) \\
& (\bar{x}(i), \bar{u}(i)) \in \mathcal{Z} \\
& \bar{x}(N)=x^{r}(N \mid k)
\end{aligned}
$$

From the optimal solution to this optimization problem $\mathbf{u}^{*}(k)$, the control law is given by $u(k)=u^{*}(0 \mid k)$.

This hierarchical control structure ensures that the controlled signal $y(k)$ converges to the best possible reachable reference $y^{r}(k)$ (according to the considered value function) if the MPC optimization problem is feasible at the initial state.

Consider the case that the signal to be tracked is changing, that is, the pattern of periodic reference signal changes with the time, leading to a transitory period of nonperiodic behavior. Then, in this case, the calculated trajectory at a certain sampling time $k$ might be not consistent with the trajectory calculated at $k-1$, that is, $x^{r}(k+$ $i \mid k-1) \neq x^{r}(k+i \mid k)$ for a certain $i$. This may render the MPC problem infeasible for the current state and the 
current reference due to the terminal constraint. Therefore, the recursive feasibility and the stability conditions of the predictive control scheme can not be guaranteed in general.

On the other hand, feasibility of the initial state is compulsory for the stability of the controller. The feasibility region depends on the prediction horizon and the reference to be tracked (due to the terminal constraint). If the reference is changed, then the feasibility region changes and the feasibility of the optimization problem at the current state may be lost.

In this paper a novel MPC is proposed to solve the tracking problem of periodic references. This controller ensures recursive feasibility, and admissible asymptotic stability of the closed loop system to the reachable trajectory. Besides the control law is calculated by solving a single optimization problem.

\section{MPC FOR TRACKING PERIODIC REFERENCES}

The proposed predictive controller combines the former two controllers in a single optimization problem, in a way such that the reachable trajectory and the sequence of future inputs are the decision variables. Thus, the predicted cost considered is the following

$$
\begin{aligned}
J\left(x, \mathbf{r}_{k} ; \overline{\mathbf{x}}^{s}, \overline{\mathbf{u}}^{s}, \overline{\mathbf{u}}\right)= & V\left(x ; \overline{\mathbf{x}}^{s}, \overline{\mathbf{u}}^{s}, \overline{\mathbf{u}}\right)+V_{p}\left(\mathbf{r}_{k} ; \overline{\mathbf{x}}^{s}, \overline{\mathbf{u}}^{s}\right) \\
= & \sum_{i=0}^{N-1}\left\|\bar{y}(i)-\bar{y}^{s}(i)\right\|_{Q}^{2}+\left\|\bar{u}(i)-\bar{u}^{s}(i)\right\|_{R}^{2} \\
& +\sum_{i=0}^{N_{r}-1}\left\|\bar{y}^{s}(i)-r(k+i)\right\|_{T}^{2}
\end{aligned}
$$

where it is assumed that $N \leq N_{r}$.

The term $V\left(x ; \mathbf{x}^{s}, \mathbf{u}^{s}, \mathbf{u}\right)$ is the cost that penalizes the tracking error with the reachable trajectory predicted for a prediction horizon $N$, while the term $V_{p}\left(\mathbf{r}_{k} ; \mathbf{x}^{s}, \mathbf{u}^{s}\right)$ penalizes the error between the reachable trajectory and the reference to be tracked predicted for one period $N_{r}$.

The control law is derived from the solution of following optimization problem

$$
\begin{array}{rl}
\min _{\overline{\mathbf{u}}, \overline{\mathbf{x}}^{s}, \overline{\mathbf{u}}^{s}} & J\left(x, \mathbf{r}_{k} ; \overline{\mathbf{x}}^{s}, \overline{\mathbf{u}}^{s}, \overline{\mathbf{u}}\right) \\
\text { s.t. } & \bar{x}(i+1)=A \bar{x}(i)+B \bar{u}(i) \\
& \bar{x}(0)=x \\
& \bar{y}(i)=C \bar{x}(i)+D \bar{u}(i) \\
& (\bar{x}(i), \bar{u}(i)) \in \mathcal{Z} \\
& \bar{x}^{s}(i+1)=A \bar{x}^{s}(i)+B \bar{u}^{s}(i) \\
& \bar{y}^{s}(i)=C \bar{x}^{s}(i)+D \bar{u}^{s}(i) \\
& \left(\bar{x}^{s}(i), \bar{u}^{s}(i)\right) \in \mathcal{Z}^{s} \\
& \bar{x}^{s}(0)=A \bar{x}^{s}\left(N_{r}-1\right)+B \bar{u}^{s}\left(N_{r}-1\right) \\
& \bar{x}(N)=\bar{x}^{s}(N)
\end{array}
$$

The set $\mathcal{Z}^{s}$ is such that $\mathcal{Z}^{s} \subseteq \mathcal{Z} \ominus B_{\epsilon}$ where $B_{\epsilon}$ is the ball of radius $\epsilon>0$. This tighter set of constraints is added for stability reasons. The optimal solution to this optimization problem is denoted $\left(\mathbf{x}^{s *}, \mathbf{u}^{s *}, \mathbf{u}^{*}\right)$ and it is assumed to be unique.

Notice that two terminal constraints have been added for stability reasons. The constraint (3i) is added to enforce that the reachable trajectory is periodic, while the constraint $(3 \mathrm{j})$ makes that the terminal state of the predicted trajectory of the plant reaches the reachable trajectory.

Since the set of constraints does not depend on the reference signal $\mathbf{r}$, there exists a set of states $\mathcal{X}_{N} \subseteq$ $\mathbb{R}^{n}$ such that the optimization is feasible iff $x \in \mathcal{X}_{N}$. The domain of attraction $\mathcal{X}_{N}$ can be read as the set of states that can admissibly reach any admissible periodic trajectory in $N$ steps.

The control law is given by

$$
u(k)=u^{*}(0 \mid k)
$$

The proposed controller ensures that the controlled system is admissibly stabilized to the optimal trajectory.

Theorem 2. Assume that the reference $\mathbf{r}$ is a periodic signal of period $N_{r}$ and $N \leq N_{r}$ then for all initial state $x(0) \in \mathcal{X}_{N}$ the proposed predictive control law ensures that the controlled system satisfies the constraints and converges to an admissible trajectory such that $V_{p}\left(\mathbf{x}_{k}, \mathbf{u}_{k}, \mathbf{r}_{k}\right)$ is minimum.

Proof. First it will be proved that the optimization problem is recursively feasible and then the convergence of the closed-loop system will be shown.

Let define

$$
\begin{aligned}
\mathbf{u}(k+1) & =\left(u^{*}(1 \mid k), \cdots, u^{*}(N-1 \mid k), u^{s *}(N \mid k)\right) \\
\mathbf{x}^{s}(k+1) & =\left(x^{s *}(1 \mid k), \cdots, x^{s *}\left(N_{r}-1 \mid k\right), x^{s *}(0 \mid k)\right) \\
\mathbf{u}^{s}(k+1) & =\left(u^{s *}(1 \mid k), \cdots, u^{s *}\left(N_{r}-1 \mid k\right), u^{s *}(0 \mid k)\right)
\end{aligned}
$$

Given that $A x^{s *}\left(N_{r}-1 \mid k\right)+B u^{s *}\left(N_{r}-1 \mid k\right)=x^{s *}(0 \mid k)$, $u^{s *}(0 \mid k)$ is an admissible control input. On the other hand, since $x(k+1)=x^{*}(1 \mid k)$, it is easy to see that $x(i \mid k+$ $1)=x(i+1 \mid k)$ for $i=1, \cdots, N-1$ and, thanks to the fact that $u(N-1 \mid k+1)=u^{s *}(N \mid k), x(N \mid k+1)=x^{s *}(N+$ $1 \mid k)=x^{s}(N \mid k+1)$. Therefore, in virtue of the feasibility of the optimal solution at $k$, the feasibility of this solution holds, and then the optimization problem will be feasible at $k+1$.

Denote

$$
\begin{aligned}
J^{*}(k)= & V\left(x(k) ; \mathbf{x}^{s *}(k), \mathbf{u}^{s *}(k), \mathbf{u}^{*}(k)\right) \\
& +V_{p}\left(\mathbf{r}_{k} ; \mathbf{x}^{s *}(k), \mathbf{u}^{s *}(k)\right) \\
J(k+1)= & V\left(x(k+1) ; \mathbf{x}^{s}(k+1), \mathbf{u}^{s}(k+1), \mathbf{u}(k+1)\right) \\
& +V_{p}\left(\mathbf{r}_{k+1} ; \mathbf{x}^{s}(k+1), \mathbf{u}^{s}(k+1)\right)
\end{aligned}
$$

Then, the proposed sequences $\left(\mathbf{u}(k+1), \mathbf{x}^{s}(k+1), \mathbf{u}^{s}(k+\right.$ 1)) satisfy

$$
\begin{aligned}
J(k+1)-J^{*}(k)= & -\left\|y(k)-y^{s}(0)\right\|_{Q}^{2}-\left\|u(k)-u^{s}(0)\right\|_{R}^{2} \\
& +\left\|y^{s}\left(N_{r}-1 \mid k+1\right)-r\left(k+N_{r}\right)\right\|_{T}^{2}
\end{aligned}
$$




$$
-\left\|y^{s *}(0 \mid k)-r(k)\right\|_{T}^{2}
$$

Given that $y^{s}\left(N_{r}-1 \mid k+1\right)=y^{s *}(0 \mid k)$ from the equality constraint and $r\left(k+N_{r}\right)=r(k)$, we have that

$$
\begin{aligned}
J(k+1)-J^{*}(k)= & -\left\|y(k)-y^{s *}(0 \mid k)\right\|_{Q}^{2} \\
& -\left\|u(k)-u^{s *}(0 \mid k)\right\|_{R}^{2}
\end{aligned}
$$

From the optimality of the solution it is derived that

$$
\begin{aligned}
J^{*}(k+1)-J^{*}(k) \leq & -\left\|y(k)-y^{s *}(0 \mid k)\right\|_{Q}^{2} \\
& -\left\|u(k)-u^{s *}(0 \mid k)\right\|_{R}^{2}
\end{aligned}
$$

Then $x(k)$ converges to $x^{s *}(0 \mid k)$ and the optimal solution $\mathbf{u}^{*}(k)$ converges to $\mathbf{u}^{s *}(k)$. In virtue of the continuity of the optimal solution w.r.t. the state and lemma 3 , it can be proved that in this situation, $\left(\mathbf{x}^{s *}(k), \mathbf{u}^{s *}(k)\right)$ is those that minimize

$$
\begin{aligned}
\min _{\overline{\mathbf{x}}^{s}, \overline{\mathbf{u}}^{s}} & V_{p}\left(\mathbf{r}_{k} ; \overline{\mathbf{x}}^{s}, \overline{\mathbf{u}}^{s}\right) \\
\text { s.t. } & \bar{x}^{s}(i+1)=A \bar{x}^{s}(i)+B \bar{u}^{s}(i) \\
& \bar{y}^{s}(i)=C \bar{x}^{s}(i)+D \bar{u}^{s}(i) \\
& \left(\bar{x}^{s}(i), \bar{u}^{s}(i)\right) \in \mathcal{Z}^{s} \\
& \bar{x}^{s}(0)=A \bar{x}^{s}\left(N_{r}-1\right)+B \bar{u}^{s}\left(N_{r}-1\right)
\end{aligned}
$$

Now the optimality lemma is proved.

Lemma 3. Let $x$ be such that the optimal solution $\left(\mathbf{u}^{*}, \mathbf{x}^{s *}, \mathbf{u}^{s *}\right)$ of the optimization problem (3) satisfy that $x^{s *}(0)=x$ and $u^{*}(j)=u^{s *}(j)$ for $j=0,1, \cdots, N-1$, then

$$
\begin{aligned}
\left(\mathbf{x}^{s *}, \mathbf{u}^{s *}\right)=\arg \min _{\overline{\mathbf{x}}^{s}, \overline{\mathbf{u}}^{s}} & V_{p}\left(\mathbf{r}_{k} ; \overline{\mathbf{x}}^{s}, \overline{\mathbf{u}}^{s}\right) \\
\text { s.t. } & \bar{x}^{s}(i+1)=A \bar{x}^{s}(i)+B \bar{u}^{s}(i) \\
& \bar{y}^{s}(i)=C \bar{x}^{s}(i)+D \bar{u}^{s}(i) \\
& \left(\bar{x}^{s}(i), \bar{u}^{s}(i)\right) \in \mathcal{Z}^{s} \\
& \bar{x}^{s}(0)=A \bar{x}^{s}\left(N_{r}-1\right)+B \bar{u}^{s}\left(N_{r}-1\right)
\end{aligned}
$$

Proof. The proof will be done by contradiction. Assume that $\left(\mathbf{x}^{s *}, \mathbf{u}^{s *}\right)$ is not the optimal trajectory. Then the optimal one, denoted as $\left(\mathbf{x}^{s o}, \mathbf{u}^{s o}\right)$, is such that $\left(\mathbf{x}^{s o}, \mathbf{u}^{s o}\right) \neq$ $\left(\mathbf{x}^{s *}, \mathbf{u}^{s *}\right)$ and

$$
V_{p}\left(\mathbf{r}_{k} ; \mathbf{x}^{s *}, \mathbf{u}^{s *}\right)>V_{p}\left(\mathbf{r}_{k} ; \mathbf{x}^{s o}, \mathbf{u}^{s o}\right)
$$

Let denote the sequences

$$
\left(\hat{\mathbf{x}}^{s}, \hat{\mathbf{u}}^{s}\right)=\beta\left(\mathbf{x}^{s *}, \mathbf{u}^{s *}\right)+(1-\beta)\left(\mathbf{x}^{s o}, \mathbf{u}^{s o}\right)
$$

where $\beta \in(0,1]$. These sequences define a reachable trajectory, that is, they are consistent with the model and admissible, in virtue of the convexity of the constraints and the linearity of the model.

Let $K_{d b}$ be the dead-beat control gain of the system and let define the sequence $\hat{u}(j)=K_{d b}\left(\hat{x}(j)-\hat{x}^{s}(j)\right)+\hat{u}^{s}(j)$ for $\hat{x}(0)=x^{s *}(0)$ and $\hat{x}(j+1)=A \hat{x}(j)+B \hat{u}(j)$. Since $N \geq n$ and since $\left(\hat{x}^{s}(j), \hat{u}^{s}(j)\right) \in \mathcal{Z}^{s} \subseteq \mathcal{Z}_{s} \ominus B_{\epsilon}$ for all $\bar{j}=0, \cdots, N_{r}-1$ and $\hat{x}(0)=x^{s *}(0)$, in virtue of the continuity of the system and the convexity of the constraints there exist a $\hat{\beta}$ such that the $\left(\hat{\mathbf{u}}, \hat{\mathbf{x}}^{s}, \hat{\mathbf{u}}^{s}\right)$ is a feasible solution of the optimization problem for all $\beta \in[\hat{\beta}, 1)$. The cost of this solution is such that
$J\left(x^{s *}(0), \mathbf{r}_{k} ; \hat{\mathbf{u}}, \hat{\mathbf{x}}^{s}, \hat{\mathbf{u}}^{s}\right)=\left\|x^{s *}(0)-\hat{x}^{s}(0)\right\|_{P}^{2}+V_{p}\left(\mathbf{r}_{k} ; \hat{\mathbf{x}}^{s}, \hat{\mathbf{u}}^{s}\right)$

where

$$
P=\sum_{j=0}^{N-1}\left(\left(A+B K_{d b}\right)^{j}\right)^{T}\left(Q+K_{d b}^{T} R K_{d b}\right)\left(A+B K_{d b}\right)^{j}
$$

From the optimality of the solution this cost is such that

$$
V_{p}\left(\mathbf{r}_{k} ; \mathbf{x}^{s *}, \mathbf{u}^{s *}\right) \leq J\left(x^{s *}(0), \mathbf{r}_{k} ; \hat{\mathbf{u}}, \hat{\mathbf{x}}^{s}, \hat{\mathbf{u}}^{s}\right)
$$

From the definition of $\left(\hat{\mathbf{x}}^{s}, \hat{\mathbf{u}}^{s}\right)$ we have that

$$
x^{s *}(0)-\hat{x}^{s}(0)=(1-\beta)\left(x^{s *}(0)-x^{s o}(0)\right)
$$

and from the convexity of $V_{p}$ we have that

$V_{p}\left(\mathbf{r}_{k} ; \hat{\mathbf{x}}^{s}, \hat{\mathbf{u}}^{s}\right) \leq \beta V_{p}\left(\mathbf{r}_{k} ; \mathbf{x}^{s *}, \mathbf{u}^{s *}\right)+(1-\beta) V_{p}\left(\mathbf{r}_{k} ; \mathbf{x}^{s o}, \mathbf{u}^{s o}\right)$

Denoting

$$
\begin{aligned}
W(\beta)= & (1-\beta)^{2}\left\|x^{s *}(0)-x^{s o}(0)\right\|_{P}^{2} \\
& +\beta V_{p}\left(\mathbf{x}^{s *}, \mathbf{u}^{s *}, \mathbf{r}\right)+(1-\beta) V_{p}\left(\mathbf{x}^{s o}, \mathbf{u}^{s o}, \mathbf{r}\right)
\end{aligned}
$$

we have that

$$
V_{p}\left(\mathbf{r}_{k} ; \mathbf{x}^{s *}, \mathbf{u}^{s *}\right) \leq J\left(x^{s *}(0), \mathbf{r}_{k} ; \hat{\mathbf{u}}, \hat{\mathbf{x}}^{s}, \hat{\mathbf{u}}^{s}\right) \leq W(\beta)
$$

From the definition of $W(\beta)$ we infer that

$$
W(1)=V_{p}\left(\mathbf{r}_{k} ; \mathbf{x}^{s *}, \mathbf{u}^{s *}\right)
$$

Taking the partial of this function we have that

$$
\begin{aligned}
\frac{\partial W}{\partial \beta}= & -2(1-\beta)\left\|x^{s *}(0)-x^{s o}(0)\right\|_{P}^{2} \\
& +V_{p}\left(\mathbf{r}_{k} ; \mathbf{x}^{s *}, \mathbf{u}^{s *}\right)-V_{p}\left(\mathbf{r}_{k} ; \mathbf{x}^{s o}, \mathbf{u}^{s o}\right)
\end{aligned}
$$

Evaluating this derivative at $\beta=1$

$$
\left.\frac{\partial W}{\partial \beta}\right|_{\beta=1}=V_{p}\left(\mathbf{r}_{k} ; \mathbf{x}^{s *}, \mathbf{u}^{s *}\right)-V_{p}\left(\mathbf{r}_{k} ; \mathbf{x}^{s o}, \mathbf{u}^{s o}\right)
$$

From the initial assumption we have that $V_{p}\left(\mathbf{r}_{k} ; \mathbf{x}^{s *}, \mathbf{u}^{s *}\right)>$ $V_{p}\left(\mathbf{r}_{k} ; \mathbf{x}^{s o}, \mathbf{u}^{s o}\right)$ and then

$$
\left.\frac{\partial W}{\partial \beta}\right|_{\beta=1}>0
$$

Then there exists a $\bar{\beta} \in[\hat{\beta}, 1)$ such that $W(\bar{\beta})<W(1)=$ $V_{p}\left(\mathbf{r}_{k} ; \mathbf{x}^{s *}, \mathbf{u}^{s *}\right)$. Summarizing we have proved that

$$
V_{p}\left(\mathbf{r}_{k} ; \mathbf{x}^{s *}, \mathbf{u}^{s *}\right) \leq W(\bar{\beta})<V_{p}\left(\mathbf{r}_{k} ; \mathbf{x}^{s *}, \mathbf{u}^{s *}\right)
$$

which is a contradiction and hence, the lemma is proved.

\section{SOME PROPERTIES OF THE PROPOSED CONTROLLER}

The proposed controller has a number of interesting properties as the following:

(i) The proposed optimization problem is a standard multi-parametric quadratic program in $\left(x, \mathbf{r}_{k}\right)$ that can be solved for a given $\left(x(k), \mathbf{r}_{k}\right)$ using specialized algorithms [Boyd and Vandenberghe, 2006]. Furthermore, exploiting the results of [Bemporad et al., 
$2002]$, the resulting control law is a piece-wise affine function of $\left(x, \mathbf{r}_{k}\right)$.

(ii) As it was illustrated in [Limon et al., 2008], the addition of the artificial reference in the MPC formulation leads to an enlargement of the domain of attraction of the predictive controller. This property can be extended to the presented controller, but enhanced. In effect, the enlargement of the domain of attraction of the proposed controller is more significant since the domain of attraction is the set of states that can reach in $N$ steps any periodic reachable signal of the system. Since a constant reference is a possible periodic reachable signal, the domain of attraction of the proposed controller is larger than the one presented in [Limon et al., 2008], but at expense of a larger number of decision variables.

(iii) The controller ensures recursive feasibility for any (possibly non-periodic) reference signal $\mathbf{r}_{\mathbf{k}}$ and if $\mathbf{r}_{k}$ converges to a periodic reference, the controller steers the system to the best possible periodic reachable signal. In effect, assume that at $k$, the expected reference (according to $\mathbf{r}_{k-1}$ ) is denoted as $\tilde{\mathbf{r}}_{k}$, but the real reference differs from the expected $\mathbf{r}_{k} \neq \tilde{\mathbf{r}}_{k}$ (due to a change of pattern in the periodic signal). Then, the optimization problem is feasible for $\mathbf{r}_{k}$ and in virtue of the continuity of the control law $\kappa_{N}\left(x(k), \mathbf{r}_{k}\right)$, if $\mathbf{r}_{k}$ converges to the expected $\tilde{\mathbf{r}}_{k}$ (that is the reference converges to a periodic signal), then $\kappa_{N}\left(x(k), \mathbf{r}_{k}\right)$ converges to $\kappa_{N}\left(x(k), \tilde{\mathbf{r}}_{k}\right)$ and hence the trajectory of the system converges to the best reachable trajectory.

This property is illustrated in the example presented in the next section.

(iv) If $\mathcal{Z}$ is compact then the control law $\kappa_{N}\left(x(k), \mathbf{r}_{k}\right)$ is also Lipschitz continuous with respect the state $x(k)$. As the model is continuous, then the closed loop system is ISS with respect to additive disturbances [Limon et al., 2009], whenever the evolution of the plant is admissible.

\section{ILLUSTRATIVE EXAMPLE}

Let consider a linear system given by the matrices

$$
A=\left[\begin{array}{cc}
0.22 & 0.44 \\
0 & 0.88
\end{array}\right], B=\left[\begin{array}{l}
1 \\
1
\end{array}\right], C=\left[\begin{array}{ll}
1 & 0
\end{array}\right], D=0
$$

subject to constraints on the inputs and the outputs as follows

$$
|u(k)| \leq 2,|y(k)| \leq 6
$$

It is known that the reference is periodic with a period $N_{r}=50$ samples. Then the proposed MPC is designed with $Q, R$ and $T$ equal to the identity matrix.

In order to illustrate the properties of the proposed controller a periodic, but changing, reference has been chosen as shown in figures 1 and 3. This reference starts with a sawtooth wave for 3 periods, then it changes to a sinusoidal wave for 3 periods and finally it changes to a constant value.
The evolution of the output together with the reference to be tracked for the case of the system without input constraints is depicted in figure 1 and the corresponding output in figure 2. It can be seen that the reference is not reachable due to the constraints on the output of the system. Notice that in the first period the signal converges to the reference which is tracked in an admissible way. However during the samples $[100,150]$ the evolution of the system changes but maintains the feasibility of the problem. This is derived from a change in the pattern of the reference signal. See that $\mathbf{r}_{k}$ for $k=100, \cdots, 150$ are not consistent due to the change to the sinusoidal wave at sample 150. The sinusoidal wave can be tracked whenever the output is admissible. In the interval $[250,300]$ the output shape changes due to the change in the pattern of the reference from a sinusoidal wave to a constant signal. Finally notice that the constant reference is tracked without error.

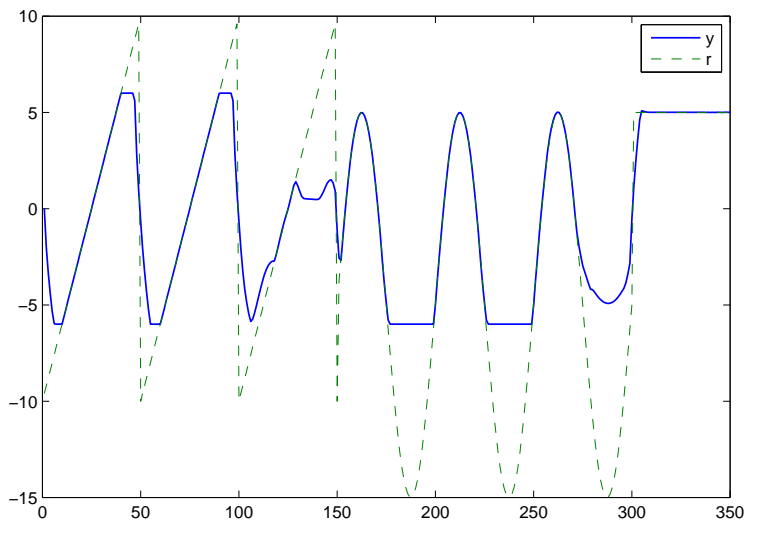

Fig. 1. Evolution of the output for the system without constraints on the inputs.

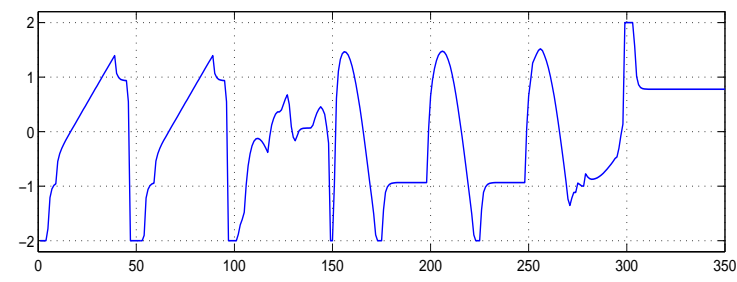

Fig. 2. Evolution of the input for the system without constraints on the inputs.

The same test has been executed considering the constraints on the inputs as $|u(k)| \leq 1$. Figures 3 and 4 show the evolution of the outputs and the inputs respectively. It can be seen that the tighter set of admissible inputs, makes that the controlled system exhibit a larger tracking error. Notice that the properties of the controller hold.

\section{CONCLUSIONS}

In this paper, the tracking problem of periodic references is considered, whose future evolution is assumed to be known but not their dynamics. This problem is analyzed for constrained linear systems and, based on the ideas of [Limon et al., 2008], a MPC controller has been proposed. 


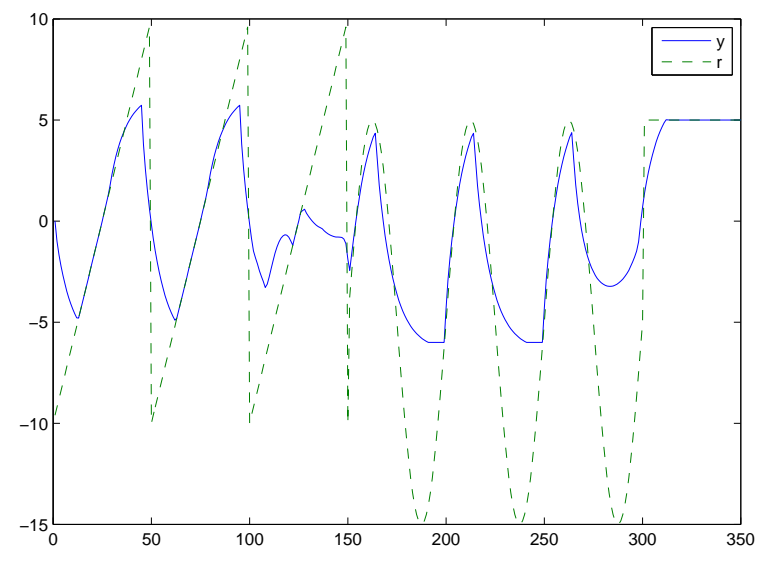

Fig. 3. Evolution of the output for the system with constraints on the inputs $|u(k)| \leq 1$.

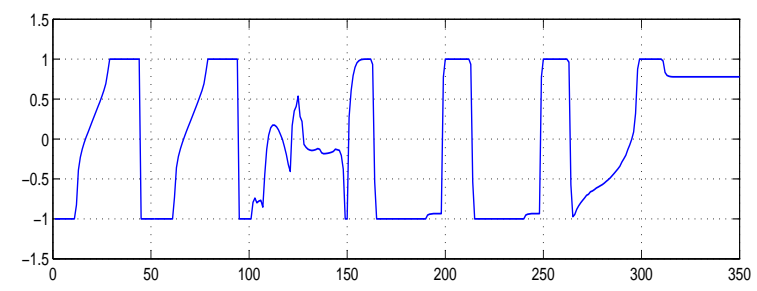

Fig. 4. Evolution of the input for the system with constraints on the inputs $|u(k)| \leq 1$.

As in [Limon et al., 2008], an artificial reachable reference is considered as decision variable as well as the predicted input of the system, a cost function that penalizes the tracking error between the predicted trajectory and the artificial reference is considered, an extended terminal constraint is added and a term penalizing the deviation between the artificial reference trajectory and the known future reference is also added. The proposed controller ensures an admissible evolution for any possible reference and asymptotic stability to the best reachable reference. Then if the reference is reachable by the constrained system, this reference will be asymptotically tracked without error.

\section{REFERENCES}

A. Bemporad, A. Casavola, and E. Mosca. Nonlinear control of constrained linear systems via predictive reference management. IEEE Transactions on Automatic Control, 42:340-349, 1997.

A. Bemporad, M. Morari, V. Dua, and E. Pistikopoulos. The explicit linear quadratic regulator for constrained systems. Automatica, 38:3-20, 2002.

S. Bittanti and P. Colaneri. Periodic Systems: Filtering and Control. Springer, 2009.

C. Böhm, T. Raff, M. Reble, and F. F. Allgöwer. Lmibased model predictive control for linear discrete-time periodic systems. Lecture Notes in Control and Information Sciences, 384, 2009.

S. Boyd and L. Vandenberghe. Convex Optimization. Cambridge University Press, 2006.

E. F. Camacho and C. Bordons. Model Predictive Control. Springer-Verlag, 2nd edition, 2004.
L. Chisci and G. Zappa. Dual mode predictive tracking of piecewise constant references for constrained linear systems. Int. J. Control, 76:61-72, 2003.

A. Ferramosca, D. Limon, I. Alvarado, T. Alamo, and E. F. Camacho. MPC for tracking with optimal closed-loop performance. Automatica, 45:1975-1978, 2009.

E. Gilbert, I. Kolmanovsky, and K. T. Tan. Discrete time reference governors and the nonlinear control of systems with state and control constraints. International Journal of Robust and Nonlinear Control, 5:487-504, 1999.

R. Gondhalekar and C.N. Jones. Mpc of constrained discrete-time linear periodic systems: A framework for asynchronous control: Strong feasibility, stability and optimality via periodic invariance. Automatica, 2:326333, 2011.

B. Kern, R. Findeisen Böhm, C., and F. Allgöwer. Receding horizon control for linear periodic time-varying systems subject to input constraints. Lecture Notes in Control and Information Sciences, 384, 2009.

K.B. Kim, J.W. Lee, and W.H. Kwon. Intervalwise receding horizon $H_{\infty}$ tracking control for discrete linear periodic systems. IEEE Transactions on Automatic Control, 45:747-752, 2000.

J.H. Lee, S. Natarajan, and K.S. Lee. A model-based predictive control approach to repetitive continuous processes with periodic operations. Journal of Process Control, 11:195-207, 2001.

D. Limon, I. Alvarado, T. Alamo, and E. F. Camacho. MPC for tracking of piece-wise constant references for constrained linear systems. Automatica, 44:2382-2387, 2008.

D. Limon, T. Alamo, D. M. Raimondo, D. Muñoz de la Peña, J. M. Bravo, A. Ferramosca, and E. F. Camacho. Input-to-state stability: an unifying framework for robust model predictive control. In L. Magni, D. M. Raimondo, and F. Allgöwer, editors, International Workshop on Assessment and Future Direction of Nonlinear Model Predictive Control, pages 1-26. Springer, 2009.

M. Lovera and A. Varga. Optimal discrete-time magnetic attitude control of satellites. In In Proc. 16th IFAC World Congress, 2005.

U. Mäder and M. Morari. Offset-Free Reference Tracking with Model Predictive Control. Automatica, 46(9): 14691476, 2010.

L. Magni, G. De Nicolao, and R. Scattolini. Output feedback and tracking of nonlinear systems with model predictive control. Automatica, 37:1601-1607, 2001.

G. Pannocchia. Robust model predictive control with guaranteed setpoint tracking. Journ. of Process Control, 14:927-937, 2004.

G. Pannocchia and E. Kerrigan. Offset-free reciding horizon control of constrained linear systems. AIChE Journal, 51:3134-3146, 2005.

J. B. Rawlings and D. Q. Mayne. Model Predictive Control: Theory and Design. Nob-Hill Publishing, 1st edition, 2009.

J. A. Rossiter, B. Kouvaritakis, and J. R. Gossner. Guaranteeing feasibility in constrained stable generalized predictive control. IEEE Proc. Control theory Appl., 143: 463-469, 1996.

A. Varga. An overview of recent developments in computational methods for periodic systems. In In Proc. IFAC workshop on periodic control systems, 2007. 